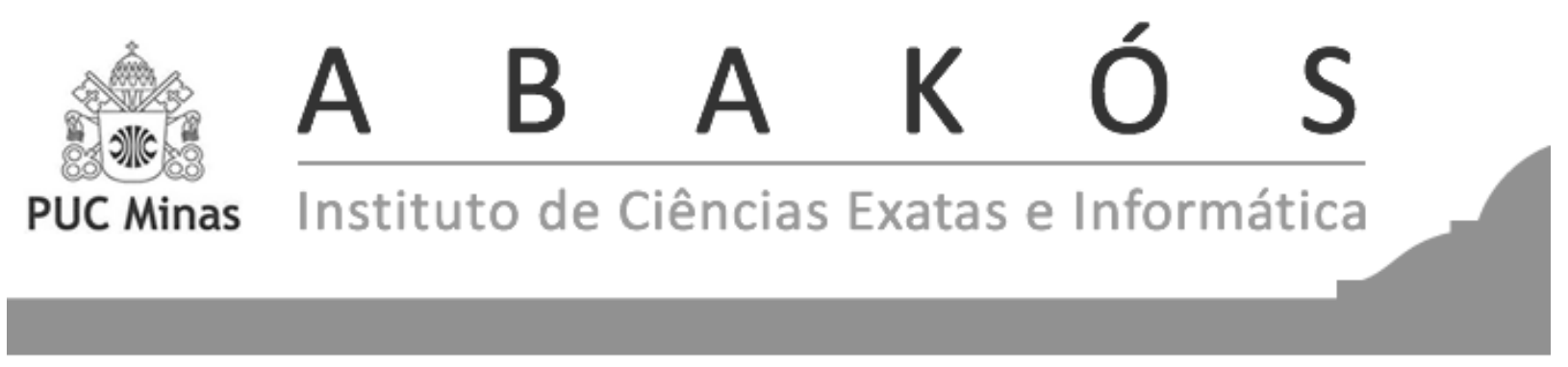

\title{
Aplicativo Shiny como Suporte de Ensino de Métodos de Previsão*
}

A Shiny Application as a Teaching Tool on Forecasting Methods

\author{
Andréa Cristina Konrath ${ }^{1}$ \\ Rodrigo Gabriel de Miranda ${ }^{2}$ \\ Luiz Ricardo Nakamura $^{1}$ \\ Elisa Henning ${ }^{3}$ \\ Olga Maria Formigoni Carvalho Walter ${ }^{4}$
}

\begin{abstract}
Resumo
Este artigo tem como objetivo apresentar uma proposta de um aplicativo Shiny com foco no ensino de métodos de previsão. O Shiny é uma ferramenta para o desenvolvimento de aplicações em web para usuários do software R, sendo um ambiente para computação estatística. O aplicativo desenvolvido contempla diversos métodos de previsão sendo eles: decomposição clássica (aditiva e multiplicativa); previsão ingênua; suavização exponencial (seleção automática; e o modelo autorregressivo integrado de médias móveis (ARIMA)), permitindo ao acadêmico importar dados de planilhas eletrônicas e produzir gráficos em uma interface amigável, gratuita e acessível. Por meio do aplicativo é possível ainda avaliar a qualidade e adequabilidade dos modelos ajustados utilizando diferentes gráficos de resíduos (dispersão, histograma, função de autocorrelação e função de autocorrelação parcial) e efetuar previsões desses ajustes a partir da TABELA 1 . Conclui-se que o desenvolvimento desse aplicativo no ambiente Shiny pode ser uma alternativa na melhoria do ensino de estatística, bem como para pesquisas aplicadas, no que tange aos métodos de previsão.
\end{abstract}

Palavras-chave: Software R. Estatística. Séries temporais.

\footnotetext{
* Submetido em 27/10/2018 - Aceito em 10/06/2019

${ }^{1}$ Departamento de Informática e Estatística-Universidade Federal de Santa Catarina, Brasil- andrea.ck@ufsc.br; luiz.nakamura@ufsc.br

${ }^{2}$ Membro do Núcleo de Normalização e Qualimetria-Universidade Federal de Santa Catarina, Brasil- rgabrieldemiranda@gmail.com

${ }^{3}$ Departamento de Matemática - Universidade do Estado de Santa Catarina, Brasil-elisa.henning@udesc.br

${ }^{4}$ Doutoranda do Programa de Pós-Graduação em Engenharia de Produção-Universidade Federal de Santa Catarina, Brasil-olgaformigoni@gmail.com
} 


\begin{abstract}
In this paper, we develop and propose a Shiny application focusing on teaching forecasting methods. Shiny is a statistical computing framework based on the software R, which is used to build several web applications. The developed application contemplates several forecasting methods namely: classical decomposition (additive and multiplicative); naive forecasting; exponential smoothing (automatic selection); and the autoregressive integrated moving average models (ARIMA), allowing the user to fit different models on data that can be imported from spreadsheets and produce different plots in a friendly, open and accessible interface. Further, we can also evaluate the fitted models through a number of different residuals plots (dispersion, histogram, autocorrelation, and partial autocorrelation functions), and produce forecasts through the application. We can conclude that the developed application, based on the Shiny framework, is an interesting alternative for the improvement of statistical teaching, as well as in applied research when we are dealing with forecasting methods.
\end{abstract}

Keywords: R software. Statistics. Time series. 


\section{INTRODUÇÃO}

O conhecimento e os avanços tecnológicos estão cada vez mais presentes no dia a dia das pessoas, logo o acesso às informações ocorre de forma cada vez mais acelerada. Nessa perspectiva, inovações tecnológicas vêm sendo inseridas no processo de ensino-aprendizagem, logo é importante que o professor utilize a tecnologia para promover esse processo. Conforme Moran (1995), a inclusão das inovações tecnológicas no ambiente educacional deve buscar a construção de conhecimentos, visando uma atuação ativa, crítica e criativa por ambas as partes: professor e aluno. O emprego das Tecnologias de Informação e Comunicação (TIC) pode tornar o processo de ensino-aprendizagem de Estatística mais eficaz e a utilização de programas estatísticos pode romper obstáculos que dificultam parte do aprendizado.

Vários autores sugerem que o ensino de disciplinas como Estatística e aquelas relacionadas à métodos quantitativos, seja apoiado por alguma ferramenta tecnológica de modo a direcionar o tempo e a atenção do aluno para a compreensão dos conceitos e aplicações, minimizando a necessidade de memorização de fórmulas e realização de cálculos manuais (SCHUYTEN; THAS, 2007; GOULD, 2010). Além disso, têm sido desenvolvidas novas orientações para o ensino superior, as quais possibilitam alternativas educacionais, que permitem reunir as modernidades da ciência e da tecnologia com o envolvimento dos alunos, tornando mais atrativos e efetivos os processos de ensino e aprendizagem (MINUTO et al., 2015).

Atualmente, na área da Estatística, um software de domínio público, livre para análise de dados, é o software R (R, 2018). O software R tem sido empregado tanto no ambiente acadêmico como no ambiente empresarial, uma vez que possui o RStudio como uma interface gráfica amigável, e o pacote Shiny como alternativa para facilitar a utilização do R via Internet. O pacote Shiny permite o desenvolvimento de aplicações para web (CHANG et al., 2017) e já dispõe de alguns trabalhos na literatura nacional (KONRATH et al., 2018; HENNING et al., 2016; MÜLLER; ZABALA, 2016). Com esse pacote, é possível transformar análises complexas em aplicações interativas e amigáveis utilizadas via web.

Neste sentido, este artigo tem como objetivo apresentar uma proposta de aplicação de métodos estatísticos de suavização exponencial com uso do pacote Shiny como apoio no ensino de métodos de previsão. O restante deste artigo está estruturado como se segue. A Seção 2 traz o referencial teórico sobre métodos de suavização exponencial. A Seção 3 descreve o que é o pacote Shiny. Os resultados obtidos com a aplicação são disponibilizados na Seção 4. Finalmente, algumas considerações finais são realizadas na Seção 5.

\section{MÉTODOS DE PREVISÃO}

A utilização de métodos de previsão no ambiente empresarial é de grande importância, pois permite que as empresas possam organizar melhor a gestão de suas atividades, bem como planejar a capacidade de seus sistemas produtivos. Com uma previsão de demanda consistente 
as empresas podem planejar as quantidades de material e mão de obra necessária para realizar suas atividades futuras. Os métodos de previsão podem ser classificados em: qualitativos e quantitativos (SLACK et al., 2009). Conforme Lemos (2006), os métodos qualitativos são fundamentados em experiências, ou seja, são modelos subjetivos. Ao passo que os métodos quantitativos, tais como os modelos causais e os de séries temporais, fazem o uso de modelos matemáticos para obter as previsões (TAKEDA, 2012). Existem vários métodos de previsão, dentre estes, se pode destacar os modelos ou métodos de suavização exponencial, que serão utilizados na aplicação Shiny proposta neste artigo.

Os métodos de suavização exponencial são uma grande classe de modelos que tentam explicar o comportamento de uma determinada variável no presente, baseada em padrões observados no passado. De maneira simplória, a ideia desse tipo de metodologia é decompor uma série temporal em componentes como, por exemplo, tendência e/ou sazonalidade, e suavizar seus valores passados, valorizando os dados mais recentes (SOUZA et al., 2008). Dentre os modelos mais comuns de suavização exponencial podem-se destacar a suavização exponencial simples (SES), para dados sem tendência e sazonalidade, o método de Holt $(\mathrm{H})$, para dados com tendência e sem sazonalidade, e o método de Holt-Winters (HW), para dados com tendência e sazonalidade.

O modelo de média móveis simples (MMS), como seu próprio nome sugere, é o mais simples possível, pois assume que todas as observações do passado possuem igual peso. Essa deficiência é corrigida nos modelos SES, em que pesos maiores são atribuídos às observações mais recentes. Entretanto, esses dois modelos não consideram os termos de tendência e sazonalidade que podem estar presentes na série temporal em estudo, componentes essas consideradas apenas nos métodos SEH e HW, respectivamente. Como esperado, quanto maior o número de detalhes considerado pelo modelo, mais complexo ele se torna. Informações mais detalhadas acerca desses métodos podem ser vistos em Morettin e Toloi (2006), Souza et al. (2008) e Hyndman e Athanasopoulos (2013).

Vale ressaltar que na classificação dos métodos de suavização exponencial deve ser levado em consideração o comportamento da tendência e da sazonalidade da série. Sendo que a tendência pode ser definida em: nenhuma, aditiva, aditiva amortecida, multiplicativa e multiplicativa amortecida, enquanto que a sazonalidade pode ser: nenhuma, aditiva e multiplicativa. Assim teremos a seguinte classificação em relação ao método: sem tendência e sem sazonalidade (NN); sem tendência e sazonalidade aditiva (NA); sem tendência e sazonalidade multiplicativa (NM); tendência aditiva e sem sazonalidade (AN); tendência aditiva e sazonalidade aditiva (AA); tendência aditiva e sazonalidade multiplicativa (AM); tendência aditiva amortecida sem sazonalidade (AdN); tendência aditiva amortecida com sazonalidade aditiva (AdA); tendência aditiva amortecida com sazonalidade multiplicativa aditiva (AdM); tendência multiplicativa sem sazonalidade (MN); tendência multiplicativa com sazonalidade aditiva (MA); tendência multiplicativa com sazonalidade multiplicativa (MM); tendência multiplicativa amortecida sem sazonalidade (MdN); tendência multiplicativa amortecida com sazonalidade aditiva $(\mathrm{MdA})$; tendência multiplicativa amortecida com sazonalidade aditiva (MdA); tendência multi- 
plicativa amortecida com sazonalidade multiplicativa (MdM), conforme a Tabela 1.

Tabela 1 - Métodos de Suavização Exponencial

\begin{tabular}{l|c|c|c}
\hline \multirow{2}{*}{ Tendência } & \multicolumn{3}{|c}{ Sazonalidade } \\
\cline { 2 - 4 } & Nenhuma & Aditiva & Multiplicativa \\
\hline Nenhuma & NN & NA & NM \\
\hline Aditiva & AN & AA & AM \\
\hline Aditiva Amortecida & AdN & AdA & AdM \\
\hline Multiplicativa & MN & MA & MM \\
\hline Multiplicativa Amortecida & MdN & MdA & MdM \\
\hline
\end{tabular}

Fonte: Souza et al. (2008).

O método NN representa uma série sem tendência e sem sazonalidade (SES). O método AN representa uma série com tendência aditiva e sem sazonalidade, denominado como o método de Holt. Já os métodos AA e AM são os métodos de Holt-Winters na forma aditiva e multiplicativa. Para cada um dos métodos existem erros, aditivos ou multiplicativos. Os símbolos e equações dos modelos podem ser vistos nas Tabelas 2 e 3 , respectivamente. Os métodos de suavização exponencial apresentados na Tabela 1 estão relacionados com a Tabela 3 , na seguinte forma: quando se têm o método sem tendência e sem sazonalidade (NN), o mesmo método está relacionado com a Equação (1) da Tabela 3. O método sem tendência e sazonalidade aditiva (NA), por exemplo, está relacionado com a Equação (2), da Tabela 3. Esse procedimento segue para os demais métodos.

Tabela 2 - Símbolos utilizados na formulação dos modelos de suavização exponencial

\begin{tabular}{c|c}
\hline Símbolo & Definição \\
\hline$y_{t}$ & Dado observado no período t \\
\hline$\alpha$ & Parâmetro de suavização para o nível \\
\hline$\beta$ & Parâmetro de suavização para o crescimento \\
\hline$\gamma$ & Parâmetro de suavização para a sazonalidade \\
\hline$\phi$ & Parâmetro de amortecimento do crescimento \\
\hline$\beta^{*}$ & $\alpha \beta$ \\
\hline$l_{t}$ & Nível da série o período t \\
\hline$b_{t}$ & Crescimento da série no período $\mathrm{t}$ \\
\hline$s_{t}$ & Sazonalidade da série o período $\mathrm{t}$ \\
\hline$F_{t+k}$ & Previsão feita no período t, k períodos à frente \\
\hline$m$ & Tamanho do ciclo sazonal \\
\hline$e_{t}$ & Erro de previsão um passo à frente \\
\hline
\end{tabular}

Fonte: Miranda et al. (2009). 
Tabela 3 - Modelos de suavização exponencial

\begin{tabular}{|c|c|c|c|}
\hline \multirow{2}{*}{ Tendência } & \multicolumn{3}{|c|}{ Sazonalidade } \\
\hline & $\mathrm{N}$ & $\bar{A}$ & $\mathrm{M}$ \\
\hline $\mathrm{N}$ & $\begin{array}{c}l_{t}=l_{t-1}+\alpha e_{t} \\
\mathrm{~F}_{t+k}=l_{t}(1)\end{array}$ & $\begin{array}{c}l_{t}=l_{t-1}+\alpha e_{t} \\
\mathrm{~s}_{t}=s_{t-m}+\gamma e_{t} \\
\mathrm{~F}_{t+k}=l_{t}+s_{t-m+k}(2)\end{array}$ & $\begin{array}{c}l_{t}=l_{t-1}+\alpha e_{t} / s_{t-m} \\
\mathrm{~s}_{t}=s_{t-m}+\gamma e_{t} / l_{t-1} \\
\mathrm{~F}_{t+k}=l_{t} s_{t-m+k}(3)\end{array}$ \\
\hline A & $\begin{array}{c}l_{t}=l_{t-1}+b_{t-1}+\alpha e_{t} \\
\mathrm{~b}_{t}=b_{t-1}+\alpha \beta e_{t} \\
\mathrm{~F}_{t+k}=l_{t}+k b_{t}(4)\end{array}$ & $\begin{array}{c}l_{t}=l_{t-1}+b_{t-1}+\alpha e_{t} \\
\mathrm{~b}_{t}=b_{t-1}+\alpha \beta e_{t} \\
\mathrm{~s}_{t}=s_{t-m}+\gamma e_{t} \\
\mathrm{~F}_{t+k}=l_{t}+k b_{t}+s_{t-m+k}(5)\end{array}$ & $\begin{array}{c}l_{t}=l_{t-1}+b_{t-1}+\alpha e_{t} / s_{t-m} \\
\mathrm{~b}_{t}=b_{t-1}+\alpha \beta e_{t} / s_{t-m} \\
\mathrm{~s}_{t}=s_{t-m}+\gamma e_{t} /\left(l_{t-1}+b_{t-1}\right) \\
\mathrm{F}_{t+k}=\left(l_{t}+k b_{t}\right) s_{t-m+k}(6)\end{array}$ \\
\hline $\mathrm{A}_{d}$ & $\begin{array}{c}l_{t}=l_{t-1}+\phi b_{t-1}+\alpha e_{t} \\
\mathrm{~b}_{t}=\phi b_{t-1}+\alpha \beta e_{t} \\
\mathrm{~F}_{t+k}=l_{t}+\sum_{i=1}^{k} \phi^{i} b_{t}(7)\end{array}$ & $\begin{array}{c}l_{t}=l_{t-1}+\phi b_{t-1}+\alpha e_{t} \\
\mathrm{~b}_{t}=\phi b_{t-1}+\alpha \beta e_{t} \\
\mathrm{~s}_{t}=s_{t-m}+\gamma e_{t} \\
\mathrm{~F}_{t+k}=l_{t}+\sum_{i=1}^{k} \phi^{i} b_{t}+s_{t-m+k}(8)\end{array}$ & $\begin{array}{c}l_{t}=l_{t-1}+\phi b_{t-1}+\alpha e_{t} / s_{t-m} \\
\mathrm{~b}_{t}=\phi b_{t-1}+\alpha \beta e_{t} / s_{t-m} \\
\mathrm{~s}_{t}=s_{t-m}+\gamma e_{t} /\left(l_{t-1}+\phi b_{t-1}\right) \\
\mathrm{F}_{t+k}=\left(l_{t}+\sum_{i=1}^{k} \phi^{i} b_{t}\right) s_{t-m+k}(9)\end{array}$ \\
\hline M & $\begin{array}{c}l_{t}=l_{t-1} b_{t-1}+\alpha e_{t} \\
\mathrm{~b}_{t}=b_{t-1}+\alpha \beta e_{t} / l_{t} \\
\mathrm{~F}_{t+k}=l_{t} b_{t}^{k}(10)\end{array}$ & $\begin{array}{c}l_{t}=l_{t-1} b_{t-1}+\alpha e_{t} \\
\mathrm{~b}_{t}=b_{t-1}+\alpha \beta e_{t} / l_{t-1} \\
\mathrm{~s}_{t}=s_{t-m}+\gamma e_{t} \\
\mathrm{~F}_{t+k}=l_{t} b_{t}^{k}+s_{t-m+k}(11)\end{array}$ & $\begin{array}{c}l_{t}=l_{t-1} b_{t-1}+\alpha e_{t} / s_{t-m} \\
\mathrm{~b}_{t}=b_{t-1}+\alpha \beta e_{t} /\left(s_{t-m} l_{t-1}\right) \\
\mathrm{s}_{t}=s_{t-m}+\gamma e_{t} /\left(l_{t-1} b_{t-1}\right) \\
\mathrm{F}_{t+k}=l_{t} b_{t}^{k} s_{t-m+k}(12)\end{array}$ \\
\hline $\mathbf{M}_{d}$ & $\begin{array}{c}l_{t}=l_{t-1} b_{t-1}^{\phi}+\alpha e_{t} \\
\mathrm{~b}_{t}=b_{t-1}^{\phi}+\alpha \beta e_{t} / l_{t-1} \\
\mathrm{~F}_{t+k}=l_{t} b_{t}^{\sum_{i=1}^{k} \phi^{i}}\end{array}$ & $\begin{array}{c}l_{t}=l_{t-1} b_{t-1}^{\phi}+\alpha e_{t} \\
\mathrm{~b}_{t}=b_{t-1}^{\phi}+\alpha \beta e_{t} / l_{t-1} \\
\mathrm{~s}_{t}=s_{t-m}+\gamma e_{t} \\
\mathrm{~F}_{t+k}=l_{t} b_{t}^{k=1} \phi^{i}+s_{t-m+k}(14)\end{array}$ & $\begin{array}{c}l_{t}=l_{t-1} b_{t-1}^{\phi}+\alpha e_{t} / s_{t-m} \\
\mathrm{~b}_{t}=b_{t-1}^{\phi}+\alpha \beta e_{t} /\left(s_{t-m} l_{t-1}\right) \\
\mathrm{s}_{t}=s_{t-m}+\gamma e_{t} /\left(l_{t-1} b_{t-1}\right) \\
\mathrm{F}_{t+k}=l_{t} b_{t}^{i=1} \phi^{i} \\
s_{t-m+k}(15)\end{array}$ \\
\hline
\end{tabular}

Fonte: Adaptado de Hyndman et al. (2008).

Métodos de Suavização exponencial são estudados por alunos de graduação em alguns cursos como Estatística, Administração e Economia, e estão presentes em vários programas de pós-graduação. Além disso, são técnicas aplicáveis em pesquisas, tanto no ensino superior como na pós-graduação. Saindo do ambiente acadêmico, também podem ser aplicadas no ambiente empresarial.

\section{AMBIENTE SHINY}

Conforme Chang et al. (2017) o pacote Shiny é um ambiente para o desenvolvimento de aplicações em web. A principal função deste pacote é permitir a comunicação entre um servidor que executa as funções do R e uma interface gráfica em HTML. A interface em HTML do aplicativo foi construída utilizando o pacote flexdashboard que permite elaborar vários layouts de páginas para visualização dos dados e gráficos (ALLAIRE, 2016). A estrutura básica de um aplicativo Shiny compreende duas partes: a primeira consiste em uma interface gráfica (UI) que possui os inputs, ou seja, os parâmetros inseridos pelo usuário e a segunda está relacionada aos outputs, que são os resultados em gráficos e tabelas. Além disso, é necessário um servidor que processa os inputs da UI no R e devolve os resultados para a mesma UI para serem exibidos nos outputs. Na Figura 1 é representada a estrutura básica de um aplicativo Shiny. 
Figura 1 - Estrutura do aplicativo

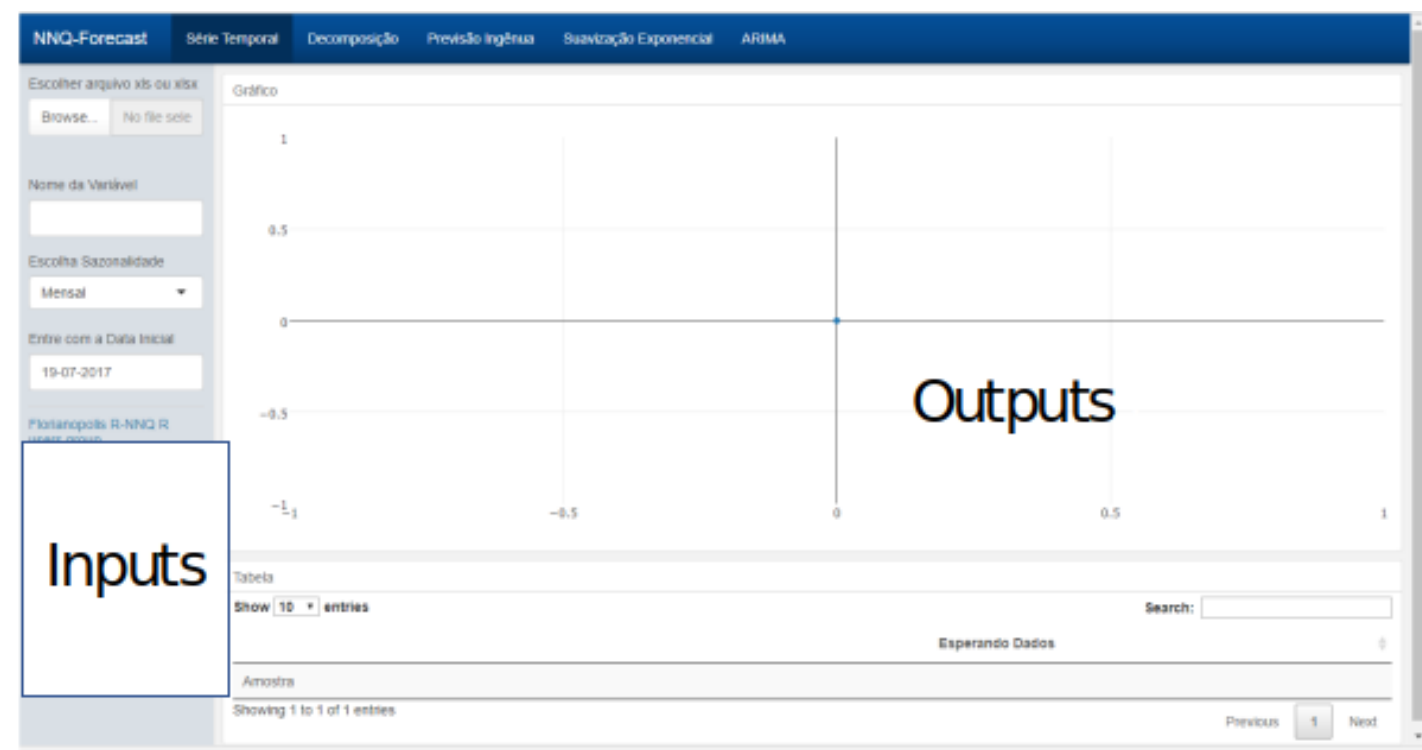

Fonte: Elaborado pelos autores

Nessa tela, os inputs do usuário são: selecionar o arquivo com os dados, selecionar a periodicidade da série e a data inicial dos dados. Os outputs são quatro gráficos: dados originais, tendência, sazonalidade e componente irregular.

Assim, o Shiny pode se configurar como uma ferramenta importante, tanto no ensino como para pesquisas aplicadas. A partir do aplicativo é possível usar algumas funções do $\mathrm{R}$ sem a necessidade de programação.

\section{APLICAÇÕES DOS MÉTODOS DE PREVISÃO POR MEIO DO SHINY}

Na elaboração do aplicativo de previsão de séries temporais foram utilizados seis pacotes do software R, cada um com uma função específica. Na Figura 2 é apresentada a tela principal da primeira versão do aplicativo desenvolvido, que pode ser acessada no seguinte link: $<$ https://estatistica.inf.ufsc.br/nnq/forecast/>. Os métodos de previsão implementados no aplicativo foram: decomposição clássica (aditiva e multiplicativa); previsão ingênua; suavização exponencial (seleção automática) e ARIMA (seleção automática). Neste artigo abordamos apenas os modelos de suavização exponencial. Informações sobre os métodos podem ser obtidas em Morettin e Toloi (2006). 


\section{Figura 2 - Aplicativo em Shiny para séries temporais}

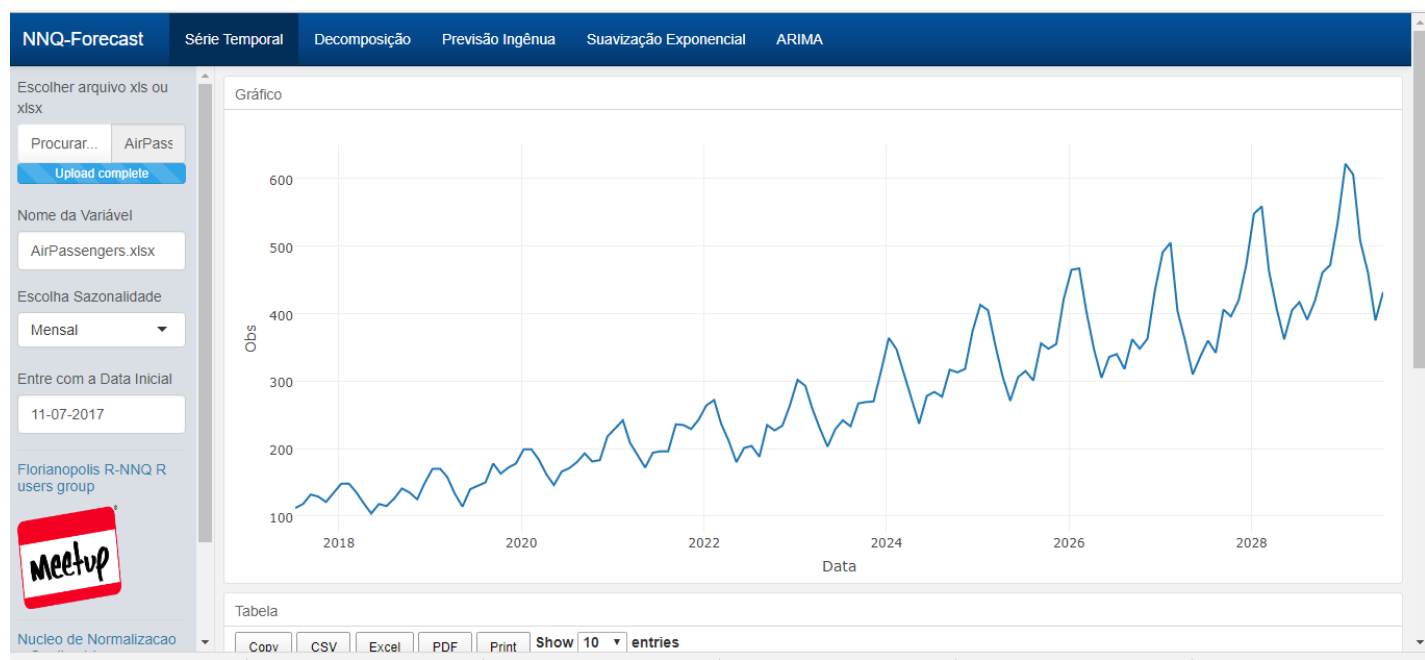

Fonte: Elaborado pelos autores

O procedimento da inserção dos dados inicia por meio da aba Série Temporal. Nessa aba o usuário irá encontrar os seguintes sidebars (Quadro 1):

\section{Quadro 1 - Sidebars do aplicativo}

\begin{tabular}{c|l|l}
\hline 1 & $\begin{array}{l}\text { Escolher arquivo com } \\
\text { extensão em xls ou xlsx }\end{array}$ & Refere-se a importação do conjunto de dados do usuário. \\
\hline 2 & Nome da variável & $\begin{array}{l}\text { Consiste no nome da variável, ou seja, variável que será } \\
\text { analisada. }\end{array}$ \\
\hline 3 & Escolha sazonalidade & $\begin{array}{l}\text { O usuário deve escolher o tipo de sazonalidade adequada: } \\
\text { diária, semanal, mensal, trimestral e anual. }\end{array}$ \\
\hline 4 & Entre com a data inicial & $\begin{array}{l}\text { O usuário deve entrar com a data inicial da série em } \\
\text { estudo. }\end{array}$ \\
\hline
\end{tabular}

Fonte: Elaborado pelos autores.

Para demonstrar o aplicativo, será apresentado um exemplo utilizando o método de suavização exponencial. O conjunto de dados consiste em uma série de dados clássica do número de passageiros por mês, compreendendo o período de 1949 a 1960 disponível no R no pacote datasets com nome AirPassengers, conforme Anexo 1 (BOX et al., 1976). Essa série apresenta tendência e sazonalidade e será utilizado o método de escolha automática de previsão, em que é escolhido o modelo com o menor erro no ajuste aos dados. Nessa tela existe a opção de escolha do modelo (todas as combinações apresentadas na Tabela 1), podendo o usuário escolher os modelos de forma individual ou automática (melhor modelo), o número de previsões, o intervalo de confiança para previsão e os gráficos para análise dos resultados. Na Figura 3 é apresentado o resultado para essa série temporal. 
Figura 3 - Resultado da série temporal

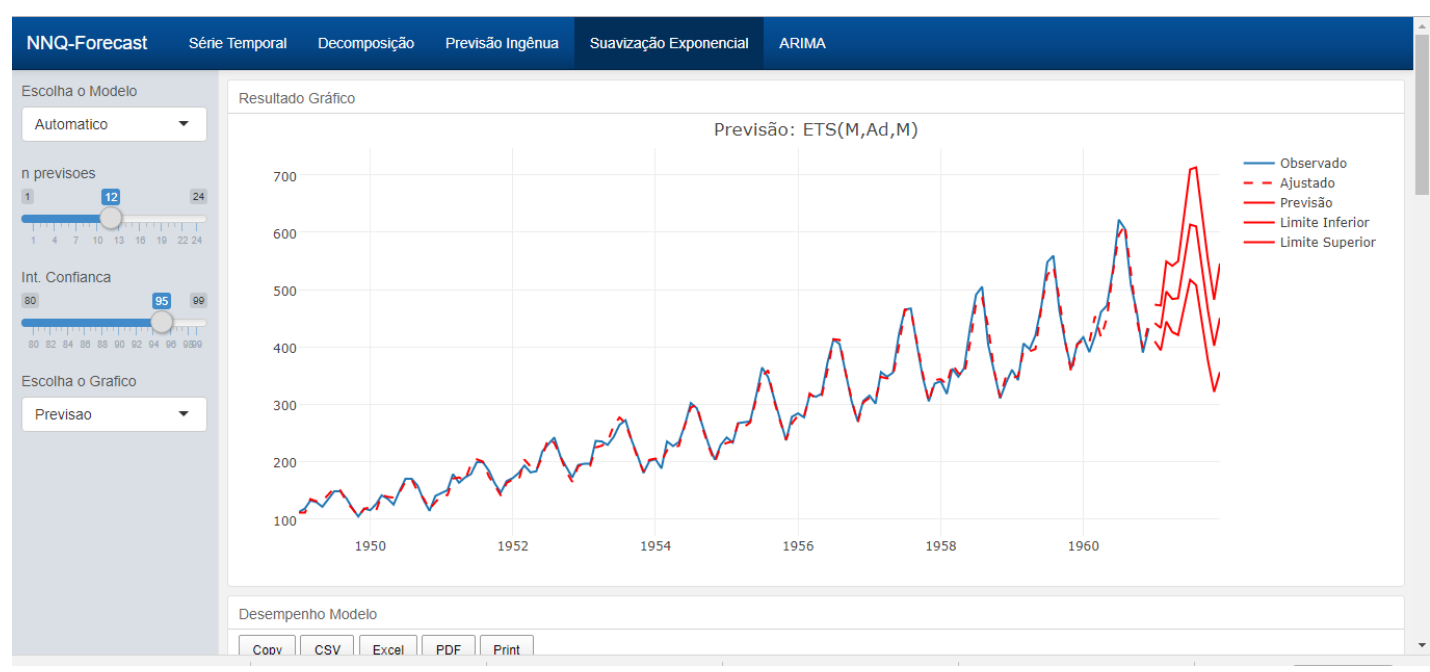

Fonte: Elaborado pelos autores

O melhor modelo encontrado foi aquele com tendência aditiva amortecida com sazonalidade multiplicativa, equações 9, conforme a Tabela 3:

$$
\begin{gathered}
l_{t}=l_{t-1}+\phi b_{t-1}+\alpha e_{t} / s_{t-m} \\
b_{t}=\phi b_{t-1}+\alpha \beta e_{t} / s_{t-m} \\
s_{t}=s_{t-m}+\gamma e_{t} /\left(l_{t-1}+\phi b_{t-1}\right) \\
F_{t+k}=\left(l_{t}+\sum_{i=1}^{k} \phi^{i} b_{t}\right) s_{t-m+k}
\end{gathered}
$$

em que são estimados o nível $\left(l_{t}\right)$, o crescimento $\left(b_{t}\right)$, a sazonalidade $\left(s_{t}\right)$ e os valores de previsão $\left(F_{t+k}\right)$. As estimativas dos parâmetros, como pode ser observado na saída do aplicativo (Figura 4), são dadas por $\hat{\alpha}=0,732, \hat{\beta}=0,019, \hat{\gamma}=0,000$ e $\hat{\phi}=0,980$ e esses valores influenciam diretamente o nível, o crescimento, a sazonalidade e o amortecimento da tendência da série temporal, respectivamente. À primeira vista, o valor do coeficiente $\hat{\gamma}$ pode causar certa estranheza, entretanto, salienta-se aqui, que ele ser nulo indica que a sazonalidade captada pelo modelo ajustado é constante ao longo de toda a série.

Figura 4 - Parâmetros do Modelo

\begin{tabular}{|l|l|l|}
\hline Parâmetros do Modelo & \\
\hline Copy CSV Excel PDF Print & \\
\hline Modelo & ETS(M,Ad,M) & \\
\hline Erro & Multiplicativo \\
\hline Tendência & Aditiva Amortecida \\
\hline Sazonalidade & Multiplicativa \\
\hline AlC & 1395.092 \\
\hline rmse & 10.668 \\
\hline alpha & 0.732 \\
\hline beta & 0.019 \\
\hline gamma & 0.000 \\
\hline phi & 0.980 \\
\hline
\end{tabular}

Fonte: Elaborado pelos autores 
Neste caso, a amplitude da sazonalidade está aumentando com crescimento na tendência da série. Na Figura 5 são apresentados os gráficos com as componentes da série.

\section{Figura 5 - Gráfico com as componentes da série temporal em estudo}

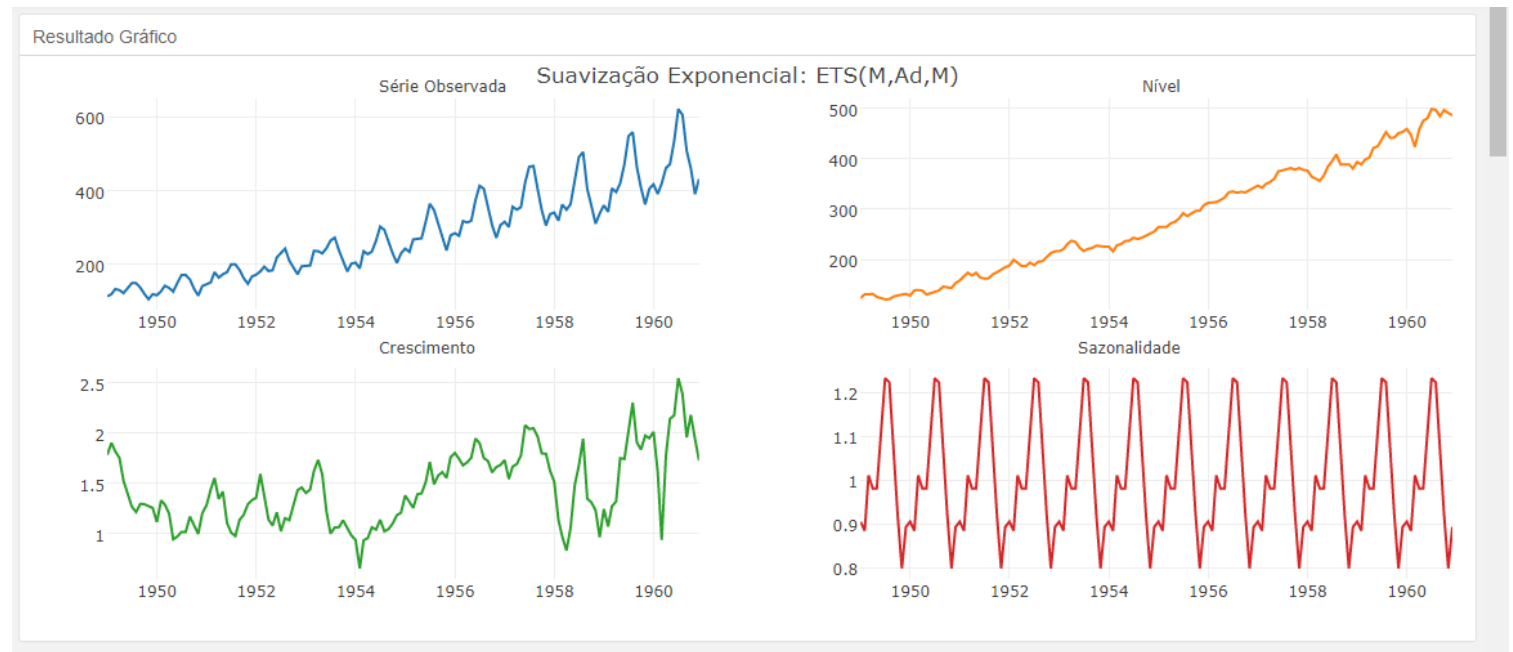

Fonte: Elaborado pelos autores

A análise da Figura 5 permite verificar que a tendência do número de passageiros aumenta de forma linear e os meses com maior sazonalidade são junho, julho e agosto (cerca de $20 \%$ maior em relação à média do ano), período de verão no hemisfério norte. Essas informações obtidas por meio dos gráficos do aplicativo desenvolvido são importantes para o planejamento de algumas atividades em uma empresa. Além dos gráficos de ajuste do modelo, o aplicativo gera gráficos com análise dos resíduos, entre eles o gráfico de dispersão, distribuição, autocorrelação e autocorrelação parcial. Esses gráficos são usados para avaliar a adequabilidade do modelo proposto e maiores detalhes podem ser consultados em Hyndman e Athanasopoulos (2013). Na Figura 6 é apresentada a dispersão dos resíduos. Verifica-se que a variância não é constante.

Figura 6 - Gráfico de dispersão dos resíduos

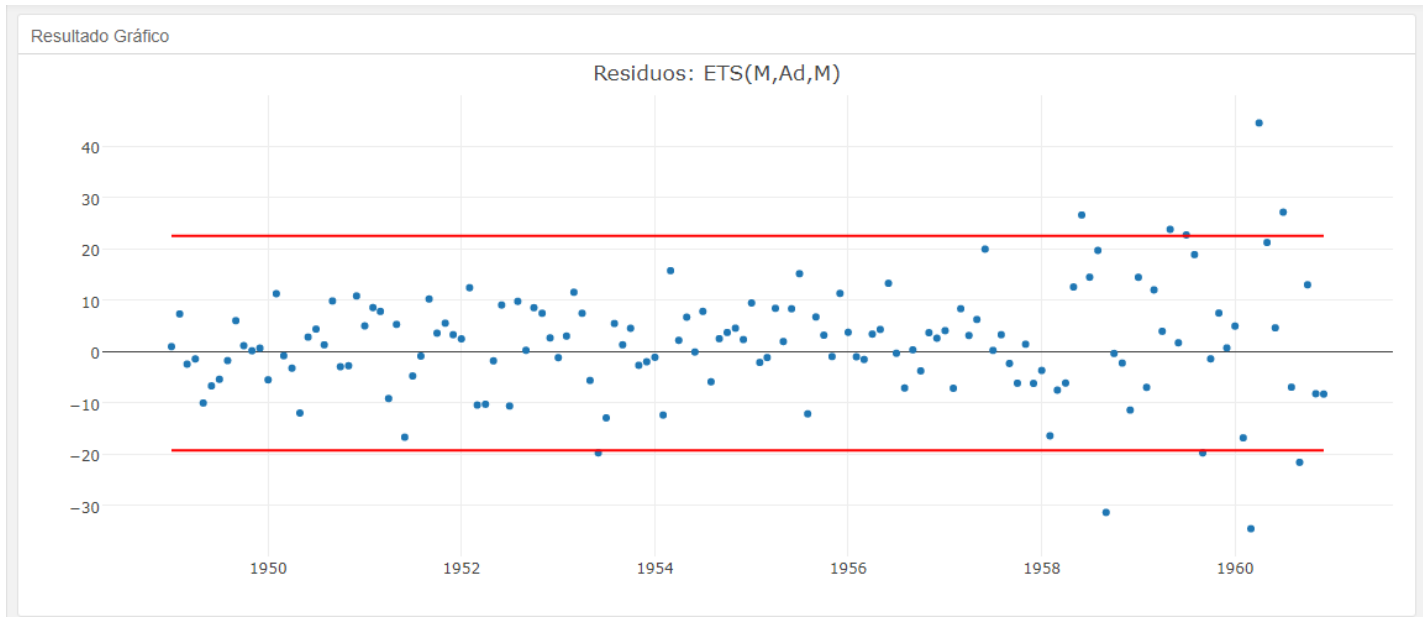

Fonte: Elaborado pelos autores 
Na Figura 7 é apresentado o gráfico de distribuição dos resíduos, que segue aproximadamente uma distribuição normal.

\section{Figura 7 - Gráfico da distribuição dos resíduos}

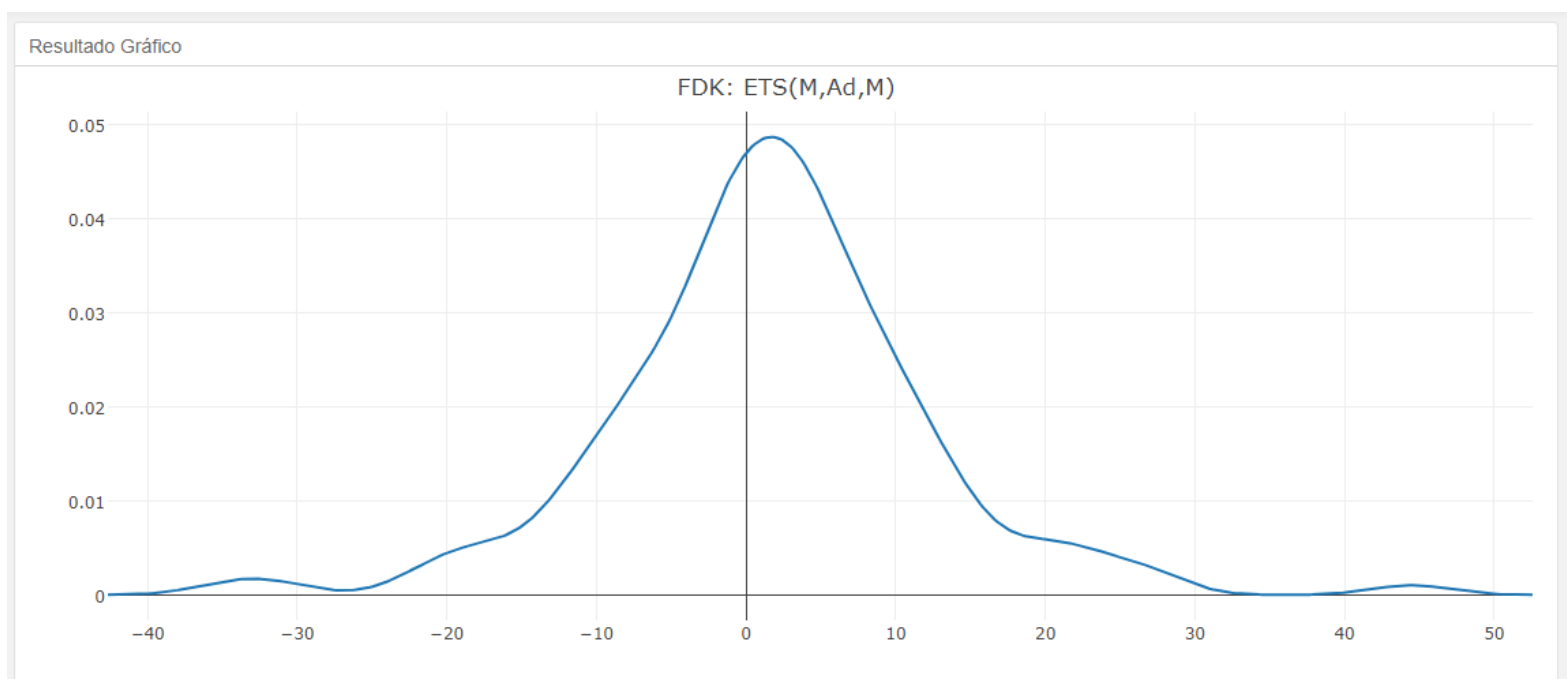

Fonte: Elaborado pelos autores

Na Figura 8 são apresentados os gráficos de autocorrelação e autocorrelação parcial.

\section{Figura 8 - Gráfico de autocorrelação e autocorrelação parcial}

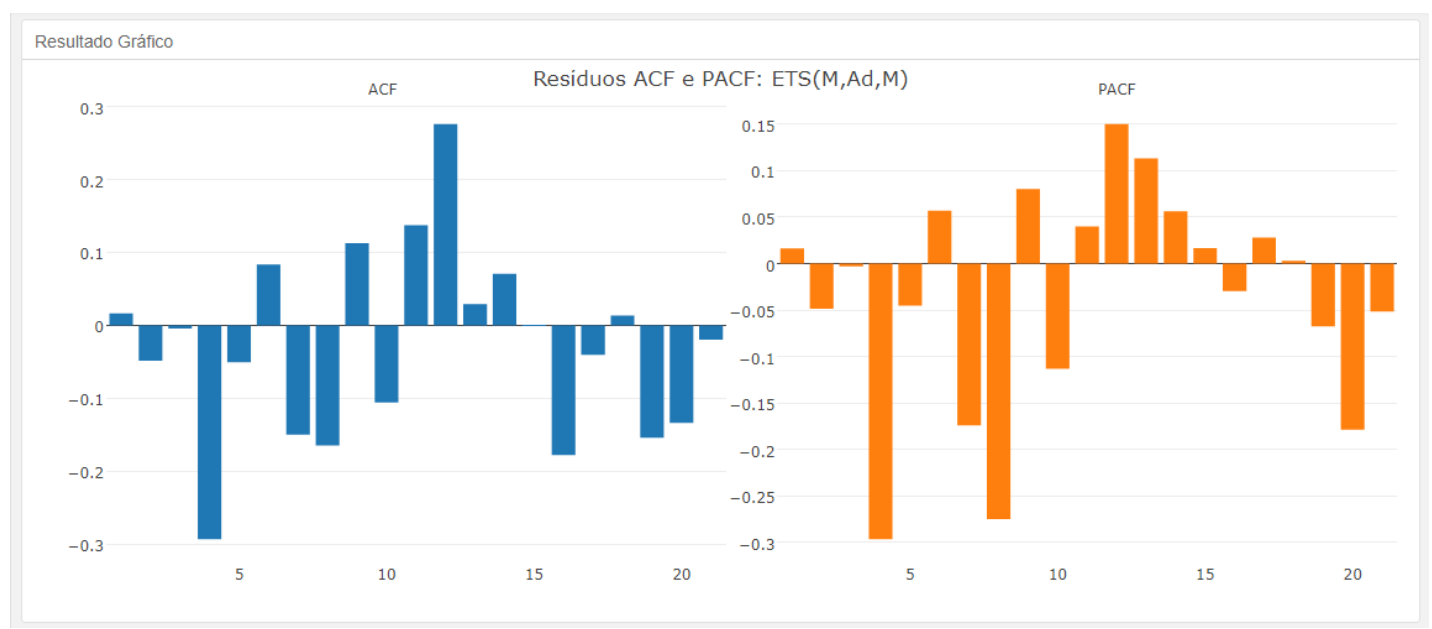

Fonte: Elaborado pelos autores

$\mathrm{O}$ aplicativo ainda necessita de aprimoramentos. Foi disponibilizado como teste em uma turma de pós-graduação em engenharia em 2018/1. O R era usado como apoio à disciplina e os alunos usavam linhas de comando para as aplicações. O uso do aplicativo foi opcional e solicitou-se dos alunos sua opinião sobre o mesmo. Uma das limitações apontadas pelos alunos foi a falta de um tutorial, ou outra forma de explicação, sobre o formato das planilhas a serem importadas. Como ponto positivo, tem-se que é um aplicativo desenvolvido em língua portuguesa, se configurando numa alternativa interessante para o uso em sala de aula. $\mathrm{Na}$ continuidade dos trabalhos recomenda-se efetuar uma aplicação em sala de aula, avaliando de forma quali-quantitativa os resultados, tanto nos aspectos de opinião quanto nos relativos ao desempenho. 
Em nossa visão, como professores, bem como educadores da área de métodos de previsão, consideramos essencial que os alunos trabalhem com aplicações a dados reais durante a disciplina. Assim, o aplicativo desenvolvido pode contribuir para tornar mais efetivo o processo de ensino aprendizagem. Ressaltamos que, não seria recomendável nos limitarmos unicamente à utilização de programas comerciais, pois o acesso fica restrito à instituição e ao período do curso.

Finalmente, ao usar aplicativos como proposto ou software livre nas atividades de ensino, nos alinhamos com o pensamento de Horton e Hardin (2015). Para os autores os dados devem ser a componente principal dos cursos de estatística. Além disso, os programas devem enfatizar conceitos e abordagens para trabalhar com dados complexos, e fornecer experiências na concepção de estudos e na análise de dados além dos livros textos. Os programas devem ser encorajados a serem criativos com seu currículo, de modo a fornecer uma síntese de teoria, dos métodos, de aspectos computacionais e aplicações.

\section{CONSIDERAÇÕES FINAIS}

O modelo tradicional de ensino utilizado nas universidades para formação de professores tem se mantido o mesmo de décadas atrás: lousa, giz, livros, trabalhos e provas. Os alunos estão ingressando nas universidades cada vez mais jovens, e o perfil do jovem estudante também é diferente do perfil de décadas atrás. Uma vez que possuem extrema facilidade para interagir com tecnologias, estão conectados quase que 24 horas em casa, no trabalho e na universidade, esses estudantes são capazes de assimilar novas informações e o uso de novas tecnologias. Nesse sentido, com a tecnologia é possível personalizar o ensino de tal modo que o aluno participe e se integre à sala de aula, absorvendo mais informações que contribuem no processo de ensinoaprendizagem.

A inclusão e o incentivo em utilizar software livre no ensino da estatística são de importância, pois contribuem no processo de ensino e aprendizagem. Uma vez que os métodos tradicionais de ensino como a simples memorização da informação não são suficientes, neste sentido buscam-se metodologias alternativas visando que processo de ensino-aprendizagem se torne mais dinâmico e interativo.

A utilização do pacote Shiny, por se tratar de um recurso computacional livre, permite que os alunos não se tornem reféns de um software comercial, e possam replicar o que foi visto em sala de aula em qualquer lugar, pois o Shiny é um aplicativo para desenvolvimento de aplicações web.

O objetivo deste artigo foi a aplicação de métodos estatísticos de suavização exponencial via pacote Shiny como apoio no ensino de métodos de previsão. Os métodos de previsão são empregados tanto no meio acadêmico como no ambiente empresarial, visto que auxiliam a tomada de decisões que, nesse último, podem estar relacionadas às áreas de controle de estoque, compras e gestão da produção, dentre outras. 
Em relação à utilização do pacote Shiny para o desenvolvimento de aplicativos interativos para a web, o mesmo se mostra uma excelente ferramenta, pois possibilita a utilização de modelos de previsão, por usuários com pouca ou nenhuma experiência de programação na linguagem R. Além disso, pela sua flexibilidade, o Shiny permite o desenvolvimento de aplicativos para outros modelos, além dos presentes no aplicativo proposto, ampliando a possibilidade do uso de modelos estatísticos. Algumas melhorias estão em andamento no aplicativo proposto como, por exemplo, a elaboração de um tutorial para usuários iniciantes e também o carregamento de dados em outros formatos. 


\section{REFERÊNCIAS}

BOX, G. E. P.; JENKINS, G. M.; REINSEL, G. C. Time series analysis forecasting and control. 3. ed. San Francisco: Holden-Day. Series G., 1976.

CHANG, W. et al. Shiny: Web Application Framework for R. R package version 1.0.0. 2017. Disponível em: <https://CRAN.R-project.org/package=shiny>. Acesso em: 07 out. 2018.

GOULD, Robert. Statistics and the modern student. International Statistical Review, Wiley Online Library, v. 78, n. 2, p. 297-315, 2010.

HENNING, E. et al. Para além da computação estatística: o uso do ambiente R para o ensino de métodos numéricos. RENOTE, v. 14, n. 1, p. 1-10, 2016.

HORTON, N.J.; HARDIN, J.S. Teaching the next generation of statistics students to "Think with Data": Special issue on statistics and the undergraduate curriculum. The American Statistician, Taylor \& Francis, v. 69, p. 259-265, 2015.

HYNDMAN, R.J.; ATHANASOPOULOS, G. Forecasting: Principles and Practice. 2013. Disponível em: <https://www.otexts.org/fpp/>. Acesso em: 15 fev. 2017.

HYNDMAN, R.J. et al. Forecasting with Exponential Smoothing: The State Space Approach. Berlin: Springer-Verlag, 2008.

KONRATH, A. C. et al. Desenvolvimento de aplicativos web com $r$ e shiny: inovações no ensino de estatística. Abakós, v. 6, n. 2, p. 55-71, 2018.

LEMOS, F.O. Metodologia para seleção de métodos de previsão de demanda. 2006. $183 \mathrm{f}$. Dissertação (Mestrado) - Escola de Engenharia, Universidade Federal do Rio Grande do Sul, Porto Alegre.

MINUTO, A.; PITTARELLO, F.; NIJHOLT, A. Smart material interfaces for education. Journal of Visual Languages \& Computing, Elsevier, v. 31, p. 267-274, 2015.

MIRANDA, R.G. et al. Espaço Admissível para os Parâmetros do Modelo de Suavização Exponencial com Dupla Sazonalidade Aditivo. 2009. 94 f. Tese (Doutorado) — Universidade Federal de Santa Catarina.

MORAN, J.M. Novas tecnologias e o reencantamento do mundo. Revista Tecnologia educacional, v. 23, n. 2, 1995.

MORETTIN, P.A.; TOLOI, C.M.C. Análise de séries temporais. 2. ed. São Paulo: Edgard Blücher, 2006.

MÜLLER, T. J.; ZABALA, F. J. Avaliação e correção automática no software livre RStudio. RENOTE, v. 14, n. 1, p. 1-10, 2016.

R, CORE TEAM. R: A language and environment for statistical computing. R Foundation for Statistical Computing. Vienna, 2018. Disponível em: <http://www.R-project.org/>. Acesso em: 08 out. 2018.

SCHUYTEN, G.; THAS, O. Statistical Thinking in Computer-Based Learning Environments. International Statistical Review, Wiley Online Library, v. 75, n. 3, p. 365-371, 2007. 
SLACK, N.; CHAMBERS, S.; JOHNSTON, R. Administração da produção. 3. ed: São Paulo: Atlas, 2009.

SOUZA, G. P.; SAMOHYL, R. W.; MIRANDA, R. G. Métodos simplificados de previsão empresarial. Rio de Janeiro: Editora Ciência Moderna, 2008.

TAKEDA, T. L. Proposta de aplicação de método quantitativo de previsão de vendas em uma indústria metal-mecânica. 2012. 71 f. Monografia (Bacharelado em Engenharia de Produção e Sistemas) — Universidade do Estado de Santa Catarina, Joinville. 
Anexo 1 - Conjunto de dados (quantidade de passageiros) - AirPassengers

\begin{tabular}{|c|c|c|c|c|c|c|c|}
\hline Seq. & Passageiros & Seq. & Passageiros & Seq. & Passageiros & Seq. & Passageiros \\
\hline $\mathbf{1}$ & 112 & $\mathbf{3 7}$ & 171 & $\mathbf{7 3}$ & 242 & $\mathbf{1 0 9}$ & 340 \\
\hline $\mathbf{2}$ & 118 & $\mathbf{3 8}$ & 180 & $\mathbf{7 4}$ & 233 & $\mathbf{1 1 0}$ & 318 \\
\hline $\mathbf{3}$ & 132 & $\mathbf{3 9}$ & 193 & $\mathbf{7 5}$ & 267 & $\mathbf{1 1 1}$ & 362 \\
\hline $\mathbf{4}$ & 129 & $\mathbf{4 0}$ & 181 & $\mathbf{7 6}$ & 269 & $\mathbf{1 1 2}$ & 348 \\
\hline $\mathbf{5}$ & 121 & $\mathbf{4 1}$ & 183 & $\mathbf{7 7}$ & 270 & $\mathbf{1 1 3}$ & 363 \\
\hline $\mathbf{6}$ & 135 & $\mathbf{4 2}$ & 218 & $\mathbf{7 8}$ & 315 & $\mathbf{1 1 4}$ & 435 \\
\hline $\mathbf{7}$ & 148 & $\mathbf{4 3}$ & 230 & $\mathbf{7 9}$ & 364 & $\mathbf{1 1 5}$ & 491 \\
\hline $\mathbf{8}$ & 148 & $\mathbf{4 4}$ & 242 & $\mathbf{8 0}$ & 347 & $\mathbf{1 1 6}$ & 505 \\
\hline $\mathbf{9}$ & 136 & $\mathbf{4 5}$ & 209 & $\mathbf{8 1}$ & 312 & $\mathbf{1 1 7}$ & 404 \\
\hline $\mathbf{1 0}$ & 119 & $\mathbf{4 6}$ & 191 & $\mathbf{8 2}$ & 274 & $\mathbf{1 1 8}$ & 359 \\
\hline $\mathbf{1 1}$ & 104 & $\mathbf{4 7}$ & 172 & $\mathbf{8 3}$ & 237 & $\mathbf{1 1 9}$ & 310 \\
\hline $\mathbf{1 2}$ & 118 & $\mathbf{4 8}$ & 194 & $\mathbf{8 4}$ & 278 & $\mathbf{1 2 0}$ & 337 \\
\hline $\mathbf{1 3}$ & 115 & $\mathbf{4 9}$ & 196 & $\mathbf{8 5}$ & 284 & $\mathbf{1 2 1}$ & 360 \\
\hline $\mathbf{1 4}$ & 126 & $\mathbf{5 0}$ & 196 & $\mathbf{8 6}$ & 277 & $\mathbf{1 2 2}$ & 342 \\
\hline $\mathbf{1 5}$ & 141 & $\mathbf{5 1}$ & 236 & $\mathbf{8 7}$ & 317 & $\mathbf{1 2 3}$ & 406 \\
\hline $\mathbf{1 6}$ & 135 & $\mathbf{5 2}$ & 235 & $\mathbf{8 8}$ & 313 & $\mathbf{1 2 4}$ & 396 \\
\hline $\mathbf{1 7}$ & 125 & $\mathbf{5 3}$ & 229 & $\mathbf{8 9}$ & 318 & $\mathbf{1 2 5}$ & 420 \\
\hline $\mathbf{1 8}$ & 149 & $\mathbf{5 4}$ & 243 & $\mathbf{9 0}$ & 374 & $\mathbf{1 2 6}$ & 472 \\
\hline $\mathbf{1 9}$ & 170 & $\mathbf{5 5}$ & 264 & $\mathbf{9 1}$ & 413 & $\mathbf{1 2 7}$ & 548 \\
\hline $\mathbf{2 0}$ & 170 & $\mathbf{5 6}$ & 272 & $\mathbf{9 2}$ & 405 & $\mathbf{1 2 8}$ & 559 \\
\hline $\mathbf{2 1}$ & 158 & $\mathbf{5 7}$ & 237 & $\mathbf{9 3}$ & 355 & $\mathbf{1 2 9}$ & 463 \\
\hline $\mathbf{2 2}$ & 133 & $\mathbf{5 8}$ & 211 & $\mathbf{9 4}$ & 306 & $\mathbf{1 3 0}$ & 407 \\
\hline $\mathbf{2 3}$ & 114 & $\mathbf{5 9}$ & 180 & $\mathbf{9 5}$ & 271 & $\mathbf{1 3 1}$ & 362 \\
\hline $\mathbf{2 4}$ & 140 & $\mathbf{6 0}$ & 201 & $\mathbf{9 6}$ & 306 & $\mathbf{1 3 2}$ & 405 \\
\hline $\mathbf{2 5}$ & 145 & $\mathbf{6 1}$ & 204 & $\mathbf{9 7}$ & 315 & $\mathbf{1 3 3}$ & 417 \\
\hline $\mathbf{2 6}$ & 150 & $\mathbf{6 2}$ & 188 & $\mathbf{9 8}$ & 301 & $\mathbf{1 3 4}$ & 391 \\
\hline $\mathbf{2 7}$ & 178 & $\mathbf{6 3}$ & 235 & $\mathbf{9 9}$ & 356 & $\mathbf{1 3 5}$ & 419 \\
\hline $\mathbf{2 8}$ & 163 & $\mathbf{6 4}$ & 227 & $\mathbf{1 0 0}$ & 348 & $\mathbf{1 3 6}$ & 461 \\
\hline $\mathbf{2 9}$ & 172 & $\mathbf{6 5}$ & 234 & $\mathbf{1 0 1}$ & 355 & $\mathbf{1 3 7}$ & 472 \\
\hline $\mathbf{3 0}$ & 178 & $\mathbf{6 6}$ & 264 & $\mathbf{1 0 2}$ & 422 & $\mathbf{1 3 8}$ & 535 \\
\hline $\mathbf{3 1}$ & 199 & $\mathbf{6 7}$ & 302 & $\mathbf{1 0 3}$ & 465 & $\mathbf{1 3 9}$ & 622 \\
\hline $\mathbf{3 2}$ & 199 & $\mathbf{6 8}$ & 293 & $\mathbf{1 0 4}$ & 467 & $\mathbf{1 4 0}$ & 606 \\
\hline $\mathbf{3 3}$ & 184 & $\mathbf{6 9}$ & 259 & $\mathbf{1 0 5}$ & 404 & $\mathbf{1 4 1}$ & 508 \\
\hline $\mathbf{3 4}$ & 162 & $\mathbf{7 0}$ & 229 & $\mathbf{1 0 6}$ & 347 & $\mathbf{1 4 2}$ & 461 \\
\hline $\mathbf{3 5}$ & 146 & $\mathbf{7 1}$ & 203 & $\mathbf{1 0 7}$ & 305 & $\mathbf{1 4 3}$ & 390 \\
\hline $\mathbf{3 6}$ & 166 & $\mathbf{7 2}$ & 229 & $\mathbf{1 0 8}$ & 336 & $\mathbf{1 4 4}$ & 432 \\
\hline
\end{tabular}

Fonte: BOX et al. (1976). 